\title{
Identification of a heterozygous ACAN mutation in a 15-year-old boy with short stature who presented with advanced bone age: a case report and review of the literature
}

Tae Youp Kim, MD', Kyung Mi Jang, MD, PhD', Chang Won Keum, $\mathrm{PhD}^{2}$, Seung Hwan Oh, MD, $\mathrm{PhD}^{3}$, Woo Yeong Chung, MD, $\mathrm{PhD}^{4}$

'Department of Pediatrics, Yeungnam University Hospital, Yeungnam University College of Medicine, Daegu, Korea ${ }^{2}$ Rare Genetic Disease Research Center, ${ }^{3}$ Billion INC, Seoul, Korea

Departments of ${ }^{3}$ Laboratory Medicine and ${ }^{4}$ Pediatrics, Inje University Busan Paik Hospital, Inje University College of Medicine, Busan, Korea

Received: 23 December, 2019

Revised: 8 February, 2020

Accepted: 18 February, 2020

Address for correspondence: Woo Yeong Chung, MD, PhD

Department of Pediatrics, Inje University Busan Paik Hospital, Inje University College of Medicine, 75 Bokji-ro, Busanjin-gu, Busan 47392, Korea

Tel: +82-51-890-6280

Fax: +82-51-897-4012,

E-mail: rarecenter@nate.com

https://orcid.org/0000-0002-50247285

Address for co-correspondence: Kyung Mi Jang, MD, PhD

Department of Pediatrics, Yeungnam University Hospital, Yeungnam University College of Medicine, 170 Hyeonchung-ro, Nam-gu, Daegu 42415, Korea

Tel: $+82-53-620-3532$

Fax: $+82-53-629-2252$

E-mail: fortune001j@gmail.com https://orcid.org/0000-0002-22269268
Longitudinal bone growth is primarily mediated by the growth plate, which is a specialized cartilaginous structure. Aggrecan, encoded by $A C A N$, is a primary proteoglycan component of the extracellular matrix in both the growth plate and articular cartilage. Aggrecanopathies have emerged as a phenotype of genetic skeletal disease in humans. A heterozygous ACAN mutation causes short stature, premature growth cessation, and accelerated bone age maturation. We report the case of a 15-year-old boy with familial short stature, with height of $149 \mathrm{~cm}$ (Korean standard deviation score [SDS] of -3.6) and weight of $50.5 \mathrm{~kg}(-1.48 \mathrm{SDS})$. He presented with mild midfacial hypoplasia, frontal bossing, a broad chest, and a short neck. The father's and mother's heights were $150 \mathrm{~cm}(-4.8$ SDS) and 153 $\mathrm{cm}(-1.69 \mathrm{SDS})$, respectively. The patient's bone age was $2-3$ years more advanced than his chronological age, and no endocrine abnormalities were detected. Wholeexome sequencing followed by Sanger sequencing revealed a heterozygous ACAN mutation, c.512C>T (p.Ala171Val), in both the proband and his father. Short stature is generally associated with a delayed bone age, and this case suggests that ACAN mutations may be the most likely etiology among patients with short stature and an advanced bone age and should warrant early treatment.

Keywords: ACAN, Aggrecan, Dysmorphism, Short stature

\section{Introduction}

Short stature in children is defined as a height of at least 2 standard deviations less than the mean for children of the same chronological age and sex. Longitudinal bone growth is primarily mediated by the growth plate, a specialized cartilaginous structure. Endochondral ossification is a complex phenomenon that promotes bone elongation and increasing height and involves proliferation, chondrocyte senescence and hypertrophy, and cartilage matrix synthesis. ${ }^{1)}$ Paracrine and autocrine factors are the primary regulators of endochondral ossification, and mutations in these genes can cause short stature. ${ }^{2)}$

Aggrecan, which is encoded by $A C A N$, is a primary proteoglycan component of the extracellular matrix of the growth plate and articular cartilage and contributes to the growth of plate cartilage. ACAN mutations were initially reported as 2 rare types of skeletal dysplasia: autosomal-dominant spondyloepiphyseal dysplasia, Kimberley type (SEDK, OMIM 608361) in 1990, ${ }^{3)}$ and autosomal-recessive spondyloepimetaphyseal dysplasia, aggrecan type (SEMD, OMIM 612813), which was previously reported in only one family. ${ }^{4)}$ A heterozygous $A C A N$ mutation has been reported as a major cause of idiopathic short stature and familial short stature. However, only one case of a young child without early advanced bone age, which is a 
typical manifestation of $A C A N$ mutation, has been reported in Korea. ${ }^{5)}$ Here, we describe the case of an adolescent boy with severely short stature who harbored a heterozygous c.512C>T (p.Ala171Val) mutation of $A C A N$, which has not yet been reported to be associated with clinical manifestation.

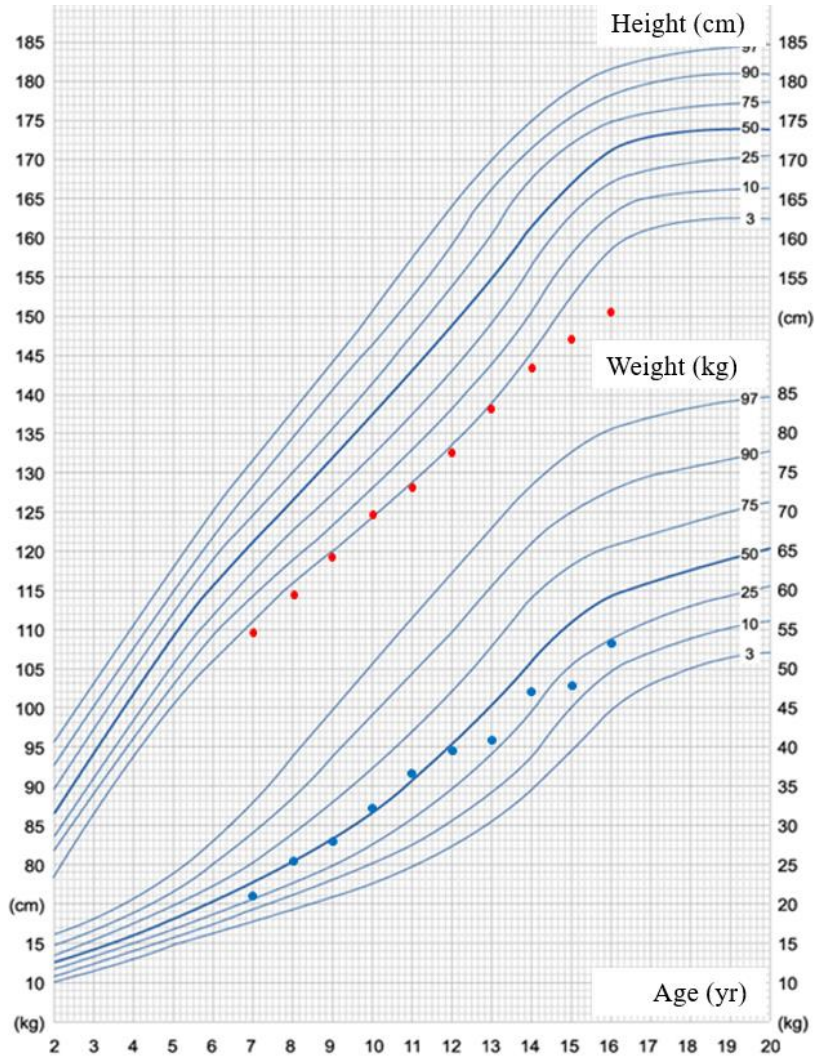

Fig. 1. The patient's height from 7-16 years of age was obtained from school physical examination records. The growth curve indicates a markedly short stature and a decreased height standard deviation score after puberty in a patient harboring an ACAN mutation.

\section{Case report}

A 15-year-old boy presented to Yeungnam University Hospital with severely short stature. He was the first of 3 children born to nonconsanguineous parents. His height and weight were $149 \mathrm{~cm}$ (Korean standard deviation score [SDS] of -3.6$)$ and $50.5 \mathrm{~kg}(-1.48 \mathrm{SDS})$, respectively. He was born at 39 weeks through normal spontaneous vaginal delivery and with a birth weight of $3.2 \mathrm{~kg}$. When we reviewed his past school physical examination records, we noted short stature and growth retardation during childhood (Fig. 1). He also presented with mild midfacial hypoplasia, frontal bossing, a broad chest, a short neck, ptosis, brachydactyly, and a low posterior hairline (Fig. 2A, B). Physical examination revealed a Tanner stage 4 and a 15- to 20-mL bilateral testicular volume. Laboratory serum analysis provided the following findings: hemoglobin, $14.6 \mathrm{~g} /$ dL; leukocytes, $9,070 / \mu \mathrm{L}$; platelet count, $341 \times 10^{3} / \mathrm{mm}$; aspartate aminotransferase, $31 \mathrm{IU} / \mathrm{L}$; alanine aminotransferase, $19 \mathrm{IU} /$ $\mathrm{L}$; sodium, $138 \mathrm{mEq} / \mathrm{L}$; potassium, $4.0 \mathrm{mEq} / \mathrm{L}$; blood urea nitrogen, $11.1 \mathrm{mg} / \mathrm{dL}$; and creatinine, $0.92 \mathrm{mg} / \mathrm{dL}$. Serum levels of insulin-like growth factor 1 and insulin-like growth factorbinding protein-3 were $486.3 \mathrm{ng} / \mathrm{mL}$ (normal range, 133-828 $\mathrm{ng} / \mathrm{mL}$ ) and $6,110 \mu \mathrm{g} / \mathrm{mL}$ (normal range, 3,287-7,735 $\mu \mathrm{g} / \mathrm{mL}$ ), respectively, and the T3, free T4, and TSH levels were $1.68 \mathrm{nmol} /$ $\mathrm{L}$ (normal range, $1.2-2.8 \mathrm{nmol} / \mathrm{L}$ ), $12.63 \mathrm{pmol} / \mathrm{L}$ (normal range, $10-20 \mathrm{pmol} / \mathrm{L}$ ), and $1.34 \mathrm{mu} / \mathrm{L}$ (normal range, $0.3-4 \mathrm{mu} / \mathrm{L}$ ). No endocrine abnormalities were detected. His bone age was 17-18 years and was determined using the standard Greulich-Pyle method, which was advanced compared with his chronological age of 15 years 3 months (Fig. 2C). Pelvic and spinal x-ray imaging did not reveal skeletal dysplasia.

His father and grandfather also had short stature. His father's and mother's heights were $150 \mathrm{~cm}(-4.8 \mathrm{SDS})$ and $153 \mathrm{~cm}(-1.69$ SDS), respectively. His younger brother and sister were 13 and 12 years old and had heights of $167 \mathrm{~cm}$ (0.36 SDS) and 157 $\mathrm{cm}(0.90$ SDS), respectively. They did not present any other abnormal clinical manifestations. The patient's father exhibited ptosis and a short neck (Fig. 2D) and had undergone pulmonary valvuloplasty at our hospital and had received treatment for
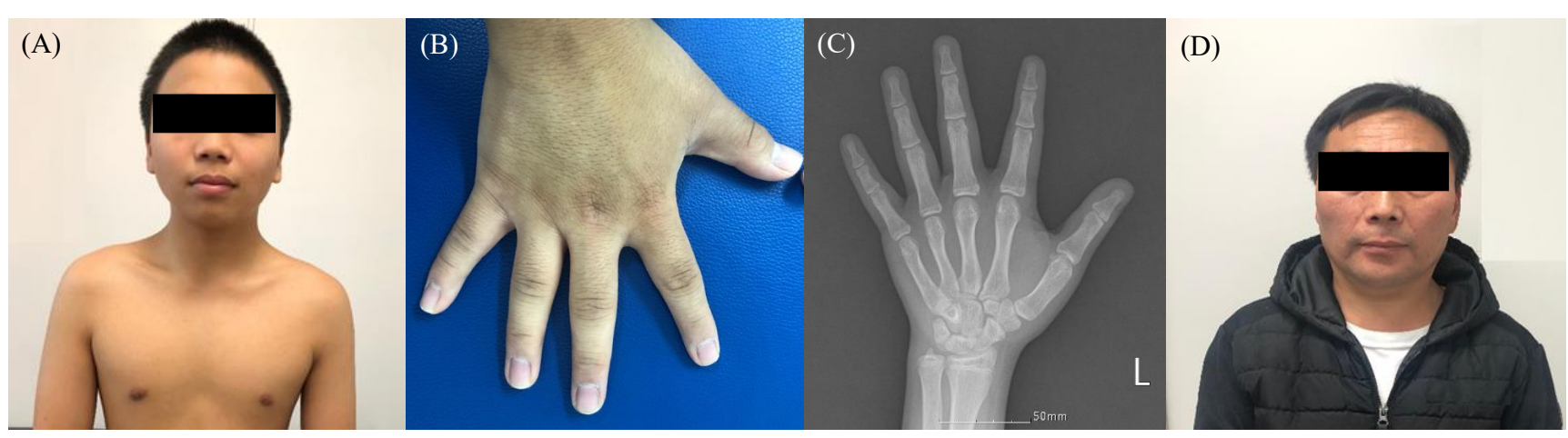

Fig. 2. Clinical and radiographic characteristics of patients harboring heterozygous ACAN mutations. (A) The patient displayed mild midfacial hypoplasia, a flat nasal bridge, and frontal bossing and (B) brachydactyly and a broad thumb. (C) The left-hand anteroposterior view represents a bone age between 17 and 18 years in accordance with the standard Greulich-Pyle method. (D) The patient's father had a short neck without midfacial hypoplasia. 
lumbar disc herniation in his 30s. Therefore, next-generation sequencing was carried out to detect Noonan syndrome; however, negative findings were obtained.

After obtaining written informed consent for genetic testing, whole-exome sequencing was performed. Genomic DNA was extracted from saliva and buccal swab samples from the patient. All exon regions of all genes $(\sim 22,000)$ were detected using the SureSelect kit (Agilent, SantaClara, CA, USA) in accordance with the manufacturer's instructions and were sequenced with the Illumina platform (NovaSeq, Illumina, San Diego, CA, USA). Data analysis for raw genome sequences (including alignment with the GRCh37/hg19 human reference genome, variant calling, and annotation) were conducted using in-house software. Variants with minor allele frequencies less than $0.05 \%$ for dominant disease associations or $2 \%$ for recessive diseases in population genome databases, including the Exome Variant Server (http://evs.gs.washington.edu/EVS/), the 1000 Genomes (http://phase3browser.1000genomes.org), and ExAC (http://exac.broadinstitute.org/), were considered for further interpretation. The proband's clinical profile was accessed to assess similarity with $\sim, 000$ genetic diseases. The pathogenicity of each variant and its associated diseases was evaluated in accordance with the ACMG/AMP guidelines without adjustment of criteria weighting. Heterozygous variant c.512C $>$ T was detected in exon 4 in ACAN (p.Ala171 Val) (Fig. 3A). This variant was considered potentially pathogenic in accordance with the PM1, PM2, PP3, and PP5 criteria. This variant is located in the link domain of $A C A N$, which is a wellestablished functional domain (PM1) that has been reported at an extremely low frequency in the aforementioned population databases (PM2); is predicted to have a deleterious effect on the gene product via multiple in silico prediction tools (DANN [score 0.9989], EIGEN [0.9592], PrimateAI [0.7016], REVEL [0.509], and MetaSVM [0.1913]) (PP3); and is registered as "likely pathogenic" in the ClinVar database (PP5).

Direct sequencing was performed for the patient, his parents, and his younger sister, and the regions of interest were determined. His mother and younger sister displayed the wild type sequence, while the heterozygous mutation was identified in his father (Fig. 3B).

\section{Discussion}

$A C A N$ is located on chromosome $15 \mathrm{q} 26.1$ and comprises 1-18 exons and the untranslated regions. ${ }^{6,7)}$ Aggrecan, which is encoded by $A C A N$, contains a $250-\mathrm{kDa}$ protein core with approximately 100 chondroitin sulfate and 30 keratan sulfate glycosaminoglycan chains. It is linked to 3 globular domains through one large domain. These globular domains regulate interactions with other components of the extracellular matrix. ${ }^{8)}$ Aggrecanopathies have emerged as a phenotype of genetic skeletal disease in humans. Aggrecan-related bone diseases include 5 clinical phenotypes: spondyloepimetaphyseal dysplasia, aggrecan type (OMIM 612813); macrocephaly with multiple epiphyseal dysplasia (OMIM 607131); spondyloepiphyseal dysplasia, Kimberley type (OMIM 608361); familial osteochondritis dissecans, short stature, and early-onset osteoarthritis (OMIM 165800); and various idiopathic short stature phenotypes. Autosomal-dominant short stature, premature growth cessation, and accelerated bone age maturation were reported in 3 families in $2014^{9)}$; one family displayed a heterozygous missense mutation (c.7064 T>C; p.Leu2355Pro), while the second family had a base-pair substitution (c.2026+1G>A) at the splice donor site on exon 10 , resulting in skip of exon 10 . The third family displayed a frameshift mutation (c.272delA). Since then, more than 100 individuals with autosomal-dominant inherited short stature have been reported as carriers of ACAN mutations. ${ }^{10)}$
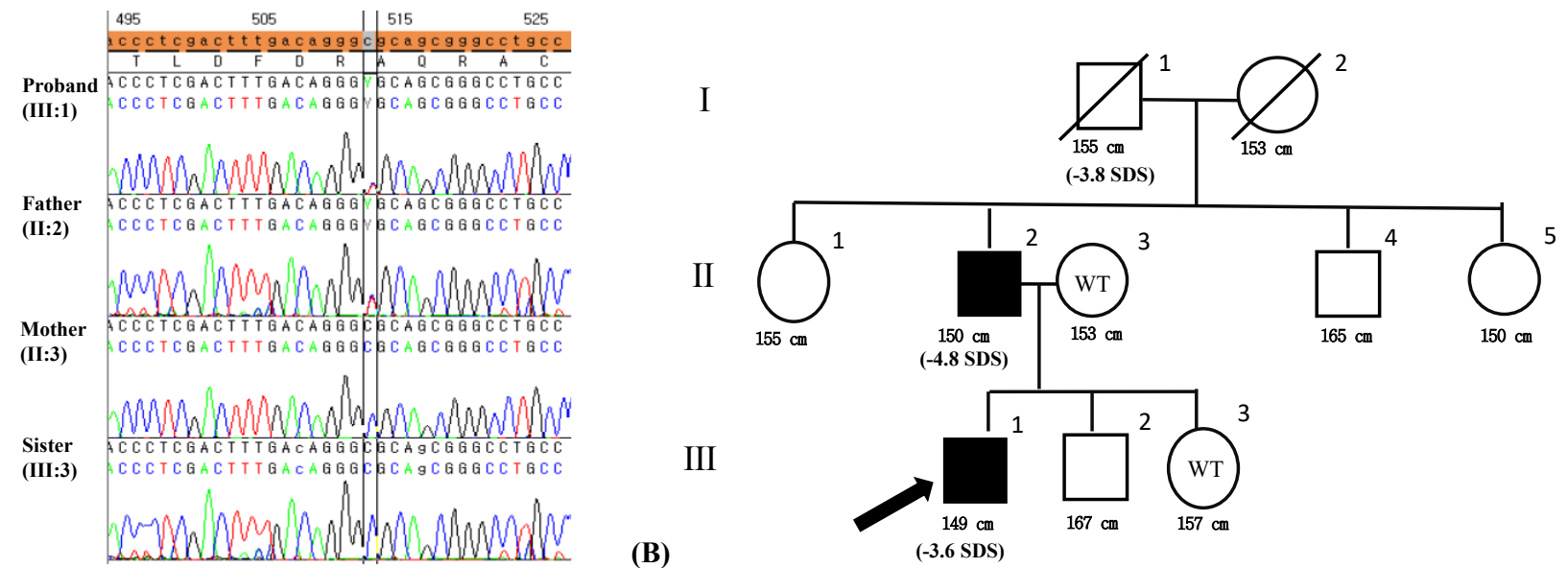

Fig. 3. Genetic testing of the family members. (A) A pedigree of the family with familial short stature. Shaded symbols indicate patients with ACAN mutations; empty symbols with a slash represent deceased members; empty symbols with "WT" are family members without mutations; empty symbols indicate family members who have not undergone ACAN sequencing. (B) The patient harbored a c.512C >T(p.Ala171Val) mutation inherited from his father. 
The phenotypic spectrum of heterozygous ACAN mutations ranges from mild and proportionate short stature to mild skeletal dysplasia with disproportionate short stature with no genotype-phenotype correlations. ACAN mutations potentially reflect mild dysmorphological findings similar to the present case, including midfacial hypoplasia, relative macrocephaly, a flat nasal bridge, frontal bossing, broad thumbs, lordosis, and brachydactyly. ${ }^{10)}$ Phenotypes may differ widely even within the same family. This phenotype spectrum suggests a dose effect with a range of penetrance. Functional aggrecan haploinsufficiency results in premature hypertrophic chondrocyte maturation and early invasion of blood vessels and osteoblasts in the growth plate, which have been proposed as a mechanism underlying advancement of bone age, premature growth cessation, and early epiphyseal fusion in patients harboring ACAN mutations. ${ }^{11,12)}$ Similar to the present case, early disc herniation is reportedly associated with $A C A N$ mutations. ${ }^{10,13)}$ A cartilage matrix-deficient mice model induced by absence of aggrecan revealed that $A C A N$ mutations result in early-onset and multiple spinal disc herniations owing to a reduction in aggrecan level in the cartilage and accelerated degeneration of disc cartilage. ${ }^{14)}$ Affected patients have advanced bone age at prepubertal stages and present with premature growth cessation after puberty. ${ }^{9,10,13)}$ Van der Steen et al. ${ }^{15)}$ reported that $A C A N$ mutations apparently influence growth impairment even before birth; these mutations were identified in a cohort study of children born small for gestational age. Gkourogianni et al. ${ }^{10)}$ reported that the median-height SDS of individuals with ACAN mutations was -2.8 in adulthood and -2.0 in childhood; and the mean difference in advanced bone age (bone age-chronologic age) was +1.3 years. Although these patients are not associated with precocious puberty, suppression of puberty with a gonadotropin releasing hormone $(\mathrm{GnRH})$ analog might be beneficial to block early growth cessation. Recent reports have indicated the effectiveness of a combined treatment that includes a $\mathrm{GnRH}$ analog and growth hormone $(\mathrm{GH})$ to help patients who harbor $A C A N$ mutations achieve an appropriate adult height. ${ }^{9,10,15)}$ A previous study reported a height increase of 5-8 cm among patients receiving combinatorial GnRH and GH treatment in comparison with sex-matched family members. ${ }^{15)}$

In summary, short stature is generally associated with delayed bone age involving GH deficiency, hypothyroidism, Cushing syndrome, and idiopathic short stature. Short stature combined with advanced bone age is much less common. Moreover, recent large-scale cohort studies of ACAN mutations have been conducted worldwide with targeted exome sequencing, and new ACAN mutations have been identified. ${ }^{16-18)}$ This case suggests that $A C A N$ mutations are the most likely etiology among patients with idiopathic short stature and advanced bone age and thus warrant early treatment.

\section{Ethical statement}

This study was approved by the Institutional Review Board (approval number: 2019-12-033) of Yeungnam University Hospital. Written informed consent was obtained from both the patient and his guardian for publication of this report.

\section{Conflict of interest}

No potential conflict of interest relevant to this article was reported.

\section{Acknowledgment}

This study was presented at the 69th Conference of the Korean Pediatric Society in 2019. We thank our patient and his family members for participation in this study.

\section{References}

1. Savendahl L. Hormonal regulation of growth plate cartilage. Horm Res 2005;64(2 Suppl):94-7.

2. Baron J, Sävendahl L, De Luca F, Dauber A, Phillip M, Wit JM, et al. Short and tall stature: a new paradigm emerges. Nat Rev Endocrinol 2015;11:735-46.

3. Anderson IJ, Tsipouras P, Scher C, Ramesar RS, Martell RW, Beighton P. Spondyloepiphyseal dysplasia, mild autosomal dominant type is not due to primary defects of type II collagen. Am J Med Genet 1990;37:272-6.

4. Tompson SW, Merriman B, Funari VA, Fresquet M, Lachman RS, Rimoin DL, et al. A recessive skeletal dysplasia, SEMD aggrecan type, results from a missense mutation affecting the C-type lectin domain of aggrecan. Am J Hum Genet 2009;84:72-9.

5. Kim YM, Cheon CK, Lim HH, Yoo HW. Identification of a novel heterozygous mutation of ACAN in a Korean family with proportionate short stature. J Genet Med 2018;15:1026.

6. Valhmu WB, Palmer GD, Rivers PA, Ebara S, Cheng JF, Fischer S, et al. Structure of the human aggrecan gene: exon-intron organization and association with the protein domains. Biochem J 1995;309:535-42.

7. Dateki S. ACAN mutations as a cause of familial short stature. Clin Pediatr Endocrinol 2017;26:119-25.

8. Roughley PJ, Mort JS. The role of aggrecan in normal and osteoarthritic cartilage. J Exp Orthop 2014;1:8.

9. Nilsson O, Guo MH, Dunbar N, Popovic J, Flynn D, Jacobsen C, et al. Short stature, accelerated bone maturation, and early growth cessation due to heterozygous aggrecan mutations. J Clin Endocrinol Metab 2014;99:1510-8.

10. Gkourogianni A, Andrew M, Tyzinski L, Crocker M, Douglas J, Dunbar N, et al. Clinical characterization of patients with autosomal dominant short stature due to aggrecan mutations. J Clin Endocrinol Metab 
2017;102:460-9.

11. Domowicz MS, Cortes M, Henry JG, Schwartz NB. Aggrecan modulation of growth plate morphogenesis. Dev Biol 2009;329:242-57.

12. Lauing KL, Cortes M, Domowicz MS, Henry JG, Baria AT, Schwartz NB. Aggrecan is required for growth plate cytoarchitecture and differentiation. Dev Biol 2014;396:224-36.

13. Dateki S, Nakatomi A, Watanabe $S$, Shimizu H, Inoue $\mathrm{Y}$, Baba $\mathrm{H}$, et al. Identification of a novel heterozygous mutation of the Aggrecan gene in a family with idiopathic short stature and multiple intervertebral disc herniation. J Hum Genet 2017;62:717-21.

14. Watanabe H, Nakata K, Kimata K, Nakanishi I, Yamada Y. Dwarfism and age-associated spinal degeneration of heterozygote $\mathrm{cmd}$ mice defective in aggrecan. Proc Natl Acad Sci U S A 1997;94:6943-7.
15. van der Steen M, Pfundt R, Maas S, Bakker-van Waarde WM, Odink RJ, Hokken-Koelega ACS. ACAN gene mutations in short children born SGA and response to growth hormone treatment. J Clin Endocrinol Metab 2017;102:1458-67.

16. Hattori A, Katoh-Fukui Y, Nakamura A, Matsubara K, Kamimaki T, Tanaka H, et al. Next generation sequencingbased mutation screening of 86 patients with idiopathic short stature. Endocr J 2017;64:947-54.

17. Hauer NN, Sticht H, Boppudi S, Buttner C, Kraus C, Trautmann U, et al. Genetic screening confirms heterozygous mutations in ACAN as a major cause of idiopathic short stature. Sci Rep 2017;7:12225.

18. Hu X, Gui B, Su J, Li H, Li N, Yu T, et al. Novel pathogenic ACAN variants in non-syndromic short stature patients. Clin Chim Acta 2017;469:126-9. 